\title{
Correspondances et contrastes entre jeux traditionnels et jeux numériques
}

Delphine Buzy-Christmann, Laurent Di Filippo, Stéphane Goria et Pauline Thévenot

\section{(2) OpenEdition}

\section{Journals}

Édition électronique

URL : http://journals.openedition.org/sdj/547

DOI : $10.4000 /$ sdj. 547

ISSN : 2269-2657

Éditeur

Laboratoire EXPERICE - Centre de Recherche Interuniversitaire Expérience Ressources Culturelles Education

\section{Référence électronique}

Delphine Buzy-Christmann, Laurent Di Filippo, Stéphane Goria et Pauline Thévenot, «

Correspondances et contrastes entre jeux traditionnels et jeux numériques », Sciences du jeu [En ligne],

5 | 2016, mis en ligne le 21 février 2016, consulté le 30 avril 2019. URL : http://

journals.openedition.org/sdj/547; DOI : 10.4000/sdj.547

Ce document a été généré automatiquement le 30 avril 2019.

Tous droits réservés 


\title{
Correspondances et contrastes entre jeux traditionnels et jeux numériques
}

\author{
Delphine Buzy-Christmann, Laurent Di Filippo, Stéphane Goria et Pauline \\ Thévenot
}

1 En 2013, au Centre National du Jeu de Boulogne-Billancourt, l'exposition Pion \& Click proposait de revenir sur les relations entre jeux de société et jeux vidéo. Pourquoi ? Parce que les liens entre ces deux types de jeux ne sont pas toujours évidents et ne renvoient pas aux mêmes représentations lorsqu'on évoque leurs pratiques. Malgré les nombreux rapports existants entre ces deux catégories de jeux, les recherches récentes centrées sur les jeux numériques ${ }^{1}$ ont souvent éclipsé celles portant sur les jeux de société et autres jeux plus "traditionnels", une des causes de ce phénomène étant l'importance des développements informatiques de ces dernières décennies. De même, alors que les premiers travaux faisant référence à l'emploi du jeu à des fins sérieuses ne se référaient pas aux jeux vidéo (Abt, 1970), il semble qu'avec l'ampleur prise par le marché des jeux vidéo, l'essentiel des études s'intéressant à l'emploi d'outils professionnels inspirés des jeux (dont l'appellation la plus connue est « serious games ») considère surtout ces outils du point de vue numérique (Alvarez \& Djaouti, 2010; Zyda, 2005). De fait, si les jeux informatisés et leurs dérivés à visée professionnelle suscitent à présent de nombreux colloques, ouvrages et numéros de revues, les réflexions sur les jeux non numériques semblent moins présentes, alors même qu'elles connaissent des développements réguliers et servent de support à la plupart des études des jeux numériques et des serious games. En effet, les réflexions anthropologiques et sociologiques menées sur les jeux par Johan Huizinga (1951) et Roger Caillois (1958) sont quasi systématiquement citées comme référence aux divers travaux proposés par les "game studies " et " serious games studies » consacrées aux applications numériques, auxquelles il faut encore ajouter les références à Jacques Henriot (1969) pour ce qui concerne surtout les recherches francophones. Dans cet ordre d'idées, les travaux portant sur les jeux numériques font aussi régulièrement référence aux réflexions portant sur les jeux "traditionnels» (non-numériques). Ce renvoi référentiel se retrouve dans de nombreux textes au niveau de la construction du 
cadre théorique (Frasca, 2001 ; Salen \& Zimmerman, 2004) ou la méthodologie de mise en œuvre d'études comparatives ou contrastives (Trémel, 2001).

Une sorte de paradoxe est en train de s'affirmer à travers la littérature scientifique en rapport avec le domaine ludique et ses applications professionnelles. Le nombre de travaux uniquement consacrés aux jeux vidéo et à leurs applications occulte petit à petit les travaux dédiés aux jeux traditionnels, alors qu'il existe toujours des liens puissants entre jeux traditionnels et jeux vidéo. Or, il nous semble évident qu'un éclairage réciproque devrait nous inciter à nous interroger sur les singularités ou complémentarités des deux domaines. C'est pourquoi nous proposons ici de nous intéresser à certains de ces liens et contrastes existants entre jeux traditionnels et jeux numériques.

3 L'objectif de cette introduction est donc de montrer une variété de problématiques qui entourent ces phénomènes sans chercher à être exhaustifs. Nous montrerons comment les articles du présent numéro, issus du colloque international « Des jeux traditionnels aux jeux numériques » (Nancy du 26 au 28 novembre 2014) contribuent à répondre à certains questionnements ouverts ici et proposent des perspectives contemporaines pour l'analyse des jeux de tous genres. Nous évoquerons aussi un certain nombre de pistes qui restent à explorer afin de sortir un peu des sentiers battus des études sur les jeux vidéo pour montrer d'autres entrées possibles. Nous mobiliserons, en outre, certains travaux parfois peu évoqués dans ce domaine d'étude pour montrer leurs possibles apports. Une place importante sera notamment faite à l'étude des rapports entre jeux vidéo et sport, rarement interrogés par les Game Studies, mais qui mérite selon nous d'être développés.

\section{Le jeu entre universalité d'une notion et particularisme des pratiques}

Vouloir trouver une origine au jeu pose de nombreux problèmes liés à la définition même que l'on donne à cette notion. Si l'on considère généralement que tous les êtres humains jouent, le jeu est alors un phénomène aussi vieux que l'humanité. Il pourrait même le précéder si l'on convient du fait que les animaux jouent eux aussi. Mais de telles perspectives sont tellement universalisantes qu'elles tiennent plutôt d'un postulat épistémologique que d'analyses concrètes. Cependant, il n'y a guère de consensus sur ces questions lorsqu'il s'agit de donner une définition plus précise des phénomènes que l'on met sous ce terme. D'un côté, ces idées se traduisent de différentes manières dans les productions scientifiques. Pour certains, le jeu serait au fondement de l'activité humaine et de la culture, comme chez Johan Huizinga et Roger Caillois qui s'attachent à comprendre les caractéristiques de l'organisation sociale (Di Filippo, 2014). Pour d'autres, l'idée de jeu est utilisée pour étudier la prise de décision comme dans la théorie des jeux de Von Neumann et Morgenstern dont s'inspirent l'économie et la sociologie, ou dans les théories de l'acteur rationnel et certains travaux d'Erving Goffman (Di Filippo, 2016). D'un autre côté, plus relativiste, on peut interroger l'universalisme d'une telle notion. Ainsi, parlant du jeu, Jacques Henriot (1989, p. 26-27) écrit qu'« il n'est pas évident qu'il y ait "quelque chose" qui corresponde à ce que conçoivent les hommes qui appartiennent à des sociétés différentes, qui vivent à des époques différentes, qui parlent des langues différentes ». Il s'agirait alors de prendre en compte les différences dans les pratiques ludiques s'inscrivant dans des contextes historiques, sociaux, culturels, géographiques, 
économiques, spécifiques, pour ne citer que quelques dimensions et de convenir, avec Patrick Schmoll (2011a, p. 10) que « les jeux accompagnent les évolutions du social et celles-ci en retour modifient la nature du jeu et de ce qui s'y joue ».

Il va sans dire que les jeux connaissent des évolutions dans le temps et dans l'espace. On pourrait ainsi multiplier les exemples. Sans citer toutes les recherches sur le sujet, on voit déjà poindre ici un problème général des recherches en sciences humaines et sociales quant à la portée des catégories d'analyse utilisées par les chercheurs. Une telle réflexion n'est donc pas propre aux sciences des jeux, mais permet de montrer comment ces dernières peuvent participer au développement plus général des sciences. Au-delà du simple aspect théorique, conceptuel ou épistémologique, il s'agit aussi de garder une certaine cohérence au triptyque objets/méthodes/théories. Cela revient également à se demander s'il est possible de développer des théories et des méthodes englobant toutes les activités ludiques ou bien si chaque objet d'étude nécessite d'adopter une démarche particulière. Est-il possible de retrouver, comme le suggérait Wittgenstein, un «air de famille» commun à tous les jeux ? Cette conception semble cependant rester un peu vague si l'on ne cherche pas qui (quel acteur) établit ces ressemblances. On peut également s'interroger à propos de la nécessité de déplacer le questionnement sur d'autres notions et s'il existe quelque chose qui serait de l'ordre du «jouable » dans les pratiques ludiques (Genvo, 2011). Comme de nombreuses notions polysémiques, les définitions du terme sont multiples et avec elles, ce sont des conceptions parfois contradictoires des phénomènes ludiques et de leur origine que l'on retrouve dans les travaux de chercheurs.

6 Par contraste, les jeux vidéo sont tenus pour un phénomène récent, ayant à peine plus d'un demi-siècle, leurs débuts étant situés à la fin des années 50 ou au début des années 60. On considère souvent Spacewar! (Steve Russell et al., 1962), dont la version arcade réalisée un peu plus tard porte le nom de Computer Space (1971: Nutting Associates) ${ }^{2}$, comme le premier jeu vidéo (Triclot, 2012), même si cela fait débat et pose la question de ce que l'on appelle jeu vidéo ainsi que les bases historiques sur lesquelles on se fonde pour définir l'antériorité comme la validité de tel jeu vis-à-vis de tel autre jeu (Baer, 2012). Certains chercheurs opposent ainsi à ce premier jeu Chase Game ${ }^{3}$ (1966), Ping Pong ${ }^{4}$ (1972 : Magnavox et Philips), Pong (1972 : Atari Inc.), Tennis for Two (1958) ou même OXO (1952) (Alvarez \& Djaouti, 2012, p. 99). Sans chercher à retracer ici l'histoire du jeu vidéo (il existe déjà de nombreux travaux traitant du sujet), nous noterons que dans la lignée d' OXO, un jeu de type Tic-Tac-Toe (ou morpion en français), les chercheurs en intelligence artificielle s'intéressèrent très tôt aux jeux de stratégie pour les programmer ${ }^{5}$. Le jeu de Nim, les Dames et le jeu d'Échecs figurent de la sorte parmi les précurseurs des jeux vidéo (Alvarez \& Djaouti, 2012). Dès les années 1940, nous recensons, notamment, les premières réflexions sur la programmation du jeu d'Échecs (Rougetet, 2015). Dans ces premières réalisations, les capacités d'affichages sont extrêmement limitées. Il est d'ailleurs intéressant de constater qu'une fois l'affichage du jeu transféré sur un écran de télévision, les premiers jeux à être développés à la suite des jeux de stratégies sont des jeux de sports (course-poursuite, tennis et ping-pong). On observe d'ores et déjà que les premiers développements des jeux vidéo s'appuient sur des éléments d'une culture ludique déjà présente. En effet, on constate facilement que des pratiques anciennes sont réajustées, modernisées et réinterprétées en fonction d'évolutions sociotechniques ou culturelles. L'industrialisation des jeux vidéo à partir des années 1970 va contribuer à une diffusion large de ces phénomènes ludiques encore nouveaux à ce moment. Pierre 
Parlebas signale, par exemple, que « de nombreux jeux, dans toutes les sociétés, sont reçus en héritage. Chaque génération transmet à la suivante un lot de pratiques ludiques qui seront acceptées et souvent réajustées aux conditions du nouveau contexte » (Parlebas, 2003, p. 6).

\section{Adaptations et filiations}

7 Un premier type d'approche qui peut être envisagé, lorsqu'on souhaite étudier les rapports entre jeux dits «traditionnels » et jeux numériques, consiste à considérer les manières dont les productions sont adaptées d'un type à l'autre en analysant leurs influences réciproques. Tout d'abord, un grand nombre de jeux non-numériques ont fait l'objet d'adaptations vidéoludiques, qu'il s'agisse d'adapter un jeu en particulier ou un titre en s'appuyant sur une franchise. Parmi les exemples de portage les plus fameux, on peut trouver des jeux de cartes, qu'ils soient directement intégrés dans les systèmes d'exploitation, en ligne ou qu'il s'agisse de jeux de cartes à collectionner (JCC), des jeux de " cafés » (comme le flipper [pinball], le billard, le bowling ou les fléchettes), des jeux de société, des jeux de rôle ou des wargames. Mais il ne faut pas oublier les jeux vidéo adaptés de jeux télévisés, comme Des chiffres et des lettres, qui a connu plusieurs adaptations, et ce dès $1987^{6}$. Ajoutons à cela différentes pratiques ludiques sportives que nous détaillerons plus bas. Les exemples ne manquent pas et montrent la variété des adaptations existantes, pouvant mener à une réflexion sur la flexibilité de l'outil numérique. Outre les besoins de diversité liés à des contraintes de marché, déjà étudiés par ailleurs, la question de la raison de l'existence de supports de multiples natures peut se poser. Il en va de même concernant l'explication de la coexistence, absence ou existence singulière de jeux de types différents sur des supports tout aussi différents, qu'ils soient vidéoludiques (de l'ordinateur aux consoles portables en passant par les bornes d'arcade ou les téléphones), électroniques (Games and Watch (1980, Nintendo), Electro (1980, Jumbo), Docteur Maboul (1965, Milton Bradley), Bataille navale (1931, Starex Novelty Co.), etc), cartonnées (jeux de plateaux et jeux de cartes), ou mixtes (Atmosfear (1991, Parker), Scene it (2005, Screenlife et Mattel), Skylanders (2011, Activision), Lord of Vermillion (2008, Square Enix), etc.). Un autre questionnement intéressant peut porter sur les règles de transformation qui s'appliquent lorsqu'un jeu non-numérique est adapté sur support numérique comme Melissa Rogerson, Martin Gibbs et Wally Smith (2015) ont pu le faire à propos de la transition jeu de plateau-jeu vidéo.

En plus de nombreux exemples de portages sur plate-forme numérique, on assiste également à de nombreux emprunts de mécaniques ludiques. Très tôt des tentatives d'adaptation de sports de raquettes, avec Tennis for Two ou Ping Pong, voient le jour. Les jeux d'action-RPG (role playing game), sans être des adaptations d'un titre spécifique, empruntent aux jeux de rôle papier des mécanismes comme les classes de personnages, la montée de niveau, ou un type de gameplay consistant au Dungeon Crawling. Quant aux jeux de stratégie, ils s'inspirent notamment des wargames sur carte ou avec figurines dont l'émancipation vidéoludique date des années 1990. Les jeux de cartes à collectionner ne sont pas en reste avec le récent succès d'Hearthstone (2014: Blizzard Entertainment). D'autres exemples mériteraient qu'on leur accorde une attention, comme les jeux de la série Mario Party (1998: Nintendo) qui reprennent certains codes du jeu de société classique : lancer de dés, plateau sur lequel les personnages avancent, convivialité par le jeu à plusieurs devant l'écran. Enfin, citons également les jeux de construction de brique 
dont s'inspirent des jeux numériques tels que Minecraft (2011 : Mojang), ou encore les jeux de piste et les chasses au trésor que l'on retrouve dans plusieurs jeux en réalité alternée $(\mathrm{ARG})^{7}$. Ces emprunts partiels montrent donc une volonté de ne pas chercher à reproduire un jeu dans sa totalité et amènent à s'interroger sur la manière dont ces décompositions et recompositions ont lieu. Pour cela, à la façon des «mythèmes » composant les récits mythiques selon Claude Levi-Strauss, les jeux pourraient être décomposés en «ludèmes ", c'est-à-dire en divers éléments constitutifs, idée que l'on retrouve également dans le discours des concepteurs de jeu contemporains (Lessard \& Therrien, 2015).

Ces nombreux exemples ne doivent pas amener à penser que la relative jeunesse des jeux vidéo conduit à ne trouver des adaptations ou des emprunts que dans un sens unique, qui irait de jeux traditionnels vers des jeux numériques. Au contraire, les jeux vidéo ou les jeux numériques ont eux aussi inspiré et donné lieu à des adaptations en diverses sortes de jeux non-numériques et de jouets. Plusieurs jeux vidéo ont eux-mêmes été adaptés en jeux de société, comme le célèbre Pac-Man (1980, Namco), Tetris (1984: The Tetris Company) et plus récemment Angry Birds (2009: Rovio Mobile). Mais ce peut être aussi l'univers du jeu qui peut-être adapté pour devenir un ou plusieurs jeux de société, comme c'est le cas pour les jeux dérivés de The Legend of Zelda (1984: Nintendo) qui ont inspiré une version du Monopoly ${ }^{8}$ ou la série World of Warcraft (2004 : Blizzard Entertainment) qui a engendré plusieurs jeux de plateau ou de cartes. Les jeux vidéo ont aussi été dérivés en des lignes de jouets, au premier rang desquels les fameux Pokémon (1996: Nintendo), mais aussi le plombier Mario (issu de Super Mario ${ }^{9}, 1985$ : Nintendo). Certains jeux vidéo furent également adaptés en jeux de rôle papier. Nous pouvons citer à titre d'exemple Dark Earth (1997 : MicroProse), adapté par la société française Multisim. Souvent, ces adaptations font suite à un certain succès commercial du jeu originel et sont le fait, avant tout, d'une exploitation marketing de l'éditeur. Elles sont néanmoins la preuve que loin de rester cantonnés à ce qu'on pourrait considérer comme une sphère vidéoludique, les jeux vidéo deviennent source d'inspiration pour d'autres types de produit et influencent la culture contemporaine de manière parfois très large (Brougère, 2008, p. 18-19).

Outre l'inspiration réciproque que suscitent les jeux traditionnels et les jeux vidéo, nous pouvons aussi nous intéresser à la question des rapports, parfois complexes, qu'entretiennent ces différentes pratiques ludiques. Par exemple, Gilles Brougère (2008, p. 6) a montré comment le concepteur du jeu Pokémon, Tajiri Satoshi, se serait inspiré des activités de la "culture ludique et traditionnelle des enfants", à savoir la collection d'insectes, à laquelle il mêle des éléments de culture ludique contemporaine comme le jouet électronique Tamagochi, ainsi que des mécanismes dérivés du jeu de rôle sur table, à savoir la montée en niveau et le combat au tour par tour. Par la suite, ce jeu vidéo fut lui même dérivé en jeu de cartes à collectionner. Nous pouvons donc en tirer une double conclusion. Premièrement, il n'existe jamais une source d'inspiration unique pour un jeu donné tout, comme il n'en existe pas pour les œuvres littéraires, et deuxièmement, un jeu inspiré de pratiques anciennes peut devenir à son tour une source pour d'autres pratiques. De même, entre deux types de productions, les rapports peuvent être variés et multiples. Ainsi, les wargames seraient à l'origine des jeux de rôles (JDR) traditionnels (qui se jouent autour d'une table) qui ont eux-mêmes inspiré les Multi Users Dungeons (MUD) et MUD, object-oriented MOO, considérés comme des prédécesseurs des MMORPG (Consalvo, 2011). Or, le MMORPG World of Warcraft (2004 : Blizzard Entertainment) s'inspire aussi de l'univers de Warcraft (1994: Blizzard Entertainment), une série de jeux de stratégie en temps réel qui emprunte aux wargames sur table avec figurines, notamment Warhammer 
Battle. On observe alors a minima des rapports de continuité double entre wargames et MMORPG. World of Warcraft donnera d'ailleurs lieu à une adaptation en JDR papier, multipliant ainsi les rapports entre différentes pratiques. Il convient alors de ne plus penser les relations entre différentes productions de manière linaire, mais d'essayer de comprendre les réseaux de relations qui se tissent entre celles-ci et qui pourraient être analysée à l'aune d'une « histoire croisée » (Werner, 2003) des pratiques ludiques.

\section{Complémentarité, développements conjoints et Transmedia}

11 Les rapports entre différentes productions ludiques, numériques et non-numériques, ne s'arrêtent cependant pas à des problématiques d'influences réciproques. En effet, les développements des outils et des technologies numériques ont aussi amené des transformations dans les pratiques de jeu traditionnelles. Le développement du web a notamment permis la mise à disposition de ressources ludiques en ligne. Des sites internet fournissent des aides de jeux, des scénarios, des fiches de personnages (la scénariothèque, le Site de l'elfe noir - SDEN, Ambiance Jeu de rôle - AJDR, etc.) pour la pratique des jeux de rôle papier et reprennent certains principes des médias non-numérique, puisque les magazines de jeux de rôle proposent généralement des scénarios et des aides de jeux. Il est possible d'établir un lien avec d'autres pratiques ludiques comme les Échecs où les magazines spécialisés tiennent une place importante en proposant des situations de jeu à résoudre (Wendling, 2002, p. 189). Ces exemples amènent alors à s'interroger sur le rôle des médias dans la constitution des pratiques ludiques et plus particulièrement ici des transformations liées aux médias numériques. Ils peuvent aussi servir à compenser la distance géographique entre joueurs, par l'usage de la visioconférence, ou d'interfaces dédiées, comme Virtuajdr ${ }^{10}$, voire simplement de programmer le calendrier des parties futures, via un forum ou des réseaux sociaux comme Facebook. Les outils numériques constituent à cet égard des supports à la vie de communauté de joueurs en établissant des relations entre les individus et un espace de performance d'un certain nombre de pratiques ludiques. Un excellent exemple ici pourrait être la chasse au trésor Sur la trace de la Chouette d'or, dont Patrick Schmoll (2007) a proposé une étude socio-ethnographique. Cet auteur montre comment les médias numériques, tout d'abord un serveur minitel, puis des forums, listes de diffusion, sites personnels et chats, participent à établir et à organiser les relations entre les joueurs participant d'un jeu dont le point de départ est un ouvrage papier regroupant les énigmes à résoudre publié en 1993 (p. 33-46). Cela permet de comprendre « les formes émergentes de sociabilité sur Internet, et à travers elles les transformations du lien social dans les sociétés qui sont les nôtres, de plus en plus fortement médiatisées par les techniques » (Schmoll, 2007, p. 11). Une partie de la pratique ludique, comme la résolution d'énigme, se déroule alors en ligne. Les espaces numériques participent dans certains cas à constituer ce que Vinciane Zabban (2014, p. 18-19) appelle le "métajeu», c'est-à-dire des " espaces de coordination et de communication ", à la fois en amont et en aval du jeu, qui influencent les manières de jouer. Et dans d'autres cas, comme pour Sur la trace de la Chouette d'or, ils sont les espaces mêmes où une partie de l'activité ludique se déroule.

12 La pédagogie peut aussi faire l'objet de croisement d'usages d'outils numériques dans des activités ludiques. Ainsi, les membres de l'association AMMS-InterESST ont mis en place des ateliers mêlant jeu de rôle et usages numériques dans l'apprentissage de l'histoire des 
sciences au lycée (Gouzouazi, Tossé, 2015, p. 261). L'objectif triple alliait à la fois le développement des capacités réflexives sur les sciences et leurs histoires, la maitrise des outils numériques et l'exercice des compétences créatives par la recherche personnelle et l'entraînement à l'exercice de rédaction. Le rôle actif des étudiants constitue le point de rencontre où s'articulent à la fois les objectifs pédagogiques de l'enseignement par "classe inversée [flipped classroom]", "où l'élève produit la connaissance de par ses recherches et ses créations" (Gouzouazi, Tossé, 2015, p. 264). C'est l'un des aspects centraux du jeu souvent mentionné par les théoriciens de cette notion, à savoir la part active du joueur dans l'activité. Ici, l'outil numérique est une composante centrale du dispositif pédagogique réflexif permettant de repenser la contextualisation sociohistorique des découvertes scientifiques en simulant une situation fictive dans laquelle un savant dispose d'un moyen de communication qui n'était pas disponible à son époque.

Récemment, on a également vu se développer des jeux que l'on pourrait qualifier d'hybrides, s'appuyant sur les technologies des écrans tactiles ou des outils de type tablette pour développer une forme de complémentarité des pratiques. Par exemple, la société E-pawn a conçu une surface électronique capable de reconnaître les objets disposant d'une puce RFID à son contact. Elle peut ainsi remplacer un plateau de jeu et réagir de façon dynamique aux figurines posées sur elle ${ }^{11}$. Dans le prolongement de cette idée d'articulation des pratiques, il faut enfin envisager les réflexions sur le «transmedia». L'usage du terme a connu d'importants développements ces dernières années et a donné lieu à des analyses aux fondements très différents. Il faut rester prudent, car les usages faits par différents auteurs de cette notion ou de notions proches ne se recoupent pas (Dena, 2009, p. 26). Chaque acception peut néanmoins s'avérer utile pour l'étude des jeux comme nous allons le voir. Ainsi, pour Jesper Juul (2005, p. 48-50), le concept de «Transmedial Games » renvoie à l'idée que les jeux se basent sur un système de règles abstrait qu'il est possible d'adapter sur différents supports. Il conviendrait alors de le suivre pour analyser différentes adaptations citées au début de notre analyse, comme les jeux de carte, de Tic-Tac-Toe/Morpion, d'Échecs, etc. Pour Lisbeth Klastrup et Susanna Tosca, l'idée de «Transmedial Worlds [monde transmedial] » correspond à l'idée abstraite d'un univers, comme celui du Seigneur des anneaux, de Marvel ou de Star Wars, qui peut être adapté sur différents supports. Simon Dor (dans ce numéro) montre comment des adaptations vidéoludiques ou sur plateau du Seigneur des anneaux sont le produit de stratégies d'adaptation différentes, dans la mesure où le récit sur lequel elles s'appuient est bien connu des joueurs. Enfin, par opposition à Klastrup et Tosca, Henry Jenkins a développé l'idée de « Transmedial storytelling [narration transmedia] ». Pour cet auteur et ceux qui s'inscrivent dans sa continuité (Bourdaa, 2012 ; Arsenault \& Mauger, 2012), c'est la complémentarité des supports qui prime sur l'œuvre originale tandis que la redondance ou la répétition nuisent à la qualité de l'ensemble des œuvres produites. Ce type de recherche permet alors d'envisager en quoi un jeu va venir compléter les connaissances sur un monde fictionnel développé dans d'autres œuvres. Enfin, on peut s'intéresser à certains jeux conçus pour être intrinsèquement hybrides et transmédiatiques comme le sont de nombreux jeux en réalité alternée. C'est ce type d'approche qu'adoptent Marida di Crosta et Ana Chantôme (dans ce numéro) en mettant en évidence le fait que l'intérêt du jeu pour ses joueurs naît aussi du flou qui existe entre les frontières médiatiques sur lesquelles le jeu se situe et se joue. 


\section{Jeu, sport et e-sport : des notions difficiles à distinguer}

Comme il a été mentionné précédemment, les sports ont inspiré certaines des premières adaptations vidéoludiques. D'ailleurs, les notions de jeu et de sport renvoient à des conceptions qu'il est souvent difficile de distinguer. Comme le résume Pierre Parlebas (2003, p. 3), " c'est peu de dire que les termes de "jeu", de "jeu traditionnel" et de "sport" sont polysémiques ; ils entretiennent de toute évidence une cascade de confusions dans tous nos discours ». D'ailleurs, chez cet auteur spécialiste du sport, l'idée de «jeu traditionnel» renvoie à des activités ludiques physiques. Sa définition de tels phénomènes est plus restreinte que celle employée dans le titre du présent article, pour lequel « jeu traditionnel » désigne simplement les jeux non-numériques dont la tradition remonte bien avant le développement des premiers jeux vidéo. Mais, comme il a été montré dans la première grande partie, ce type de catégorisations doit être nuancé au regard d'études de cas spécifiques.

15 À ce propos, il est intéressant de constater que le vocabulaire courant consacre l'expression «Jeux Olympiques » à des épreuves sportives nécessitant un entrainement rigoureux, voire professionnel, plutôt qu'à l'idée générale que l'on peut se faire d'un jeu comme participant du temps de loisiri ${ }^{12}$. Lorsque l'on parle de Jeux Olympiques, il est alors question de sport et de compétition. Or, des auteurs de références comme Huizinga et Caillois ont fait remarquer que l'aspect compétitif, désigné par le terme agôn, est un des traits fondamentaux du jeu. Dès lors, si la notion de sport s'écarte de celle de jeu, ce n'est pas tant à partir de ce point, qu'en fonction du fait que les sports dits Olympiques sont sérieux et que, depuis 1984, ces compétitions sont officiellement l'affaire d'athlètes ouvertement professionnels alors que pendant très longtemps les participants se devaient d'être de purs amateurs. De ce point de vue, il ne s'agit plus d'activités ludiques, au sens de Caillois, au moins, car on ne peut plus les caractériser de libres et de fictives. Il faut néanmoins rester prudent quant à l'usage de termes contemporains pour désigner des phénomènes anciens, puisque, comme le dit Paul Veyne (1987, p. 53), entre ce que nous désignons aujourd'hui par Jeux Olympiques et ce que les Grecs anciens appelaient

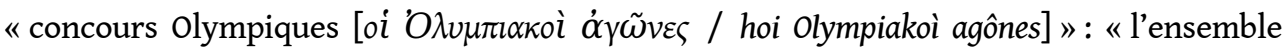
diffère, et tout le contexte ». Bien que nous ne puissions le faire ici, il serait nécessaire d'aborder cette question sous l'angle d'une histoire des catégories de pensée.

Inversement, pour peu que l'on n'impose pas comme élément fondamental du sport, la nécessité d'une dépense d'énergie liée à un effort physique, nous trouvons facilement un certain nombre de jeux de plateau (Échecs ou Go) ou de cartes (Poker) qui sont considérés comme des sports par certaines institutions ou par certains acteurs sociaux. À titre d'exemple, les Échecs sont considérés comme tels par le Comité international Olympique (CIO) depuis 1999 (Vieille Marchiset et Wendling, 2010). C'est donc le regard porté sur une pratique qui va permettre de la juger comme un sport ou un jeu. Par conséquent, il ne s'agit pas d'une question de support. Dans une optique qui se voudrait plus anthropologique et empirique, il conviendrait plutôt d'analyser pourquoi et comment les Échecs sont venus à être considérés comme un sport et les changements que cela implique. Cela permettrait d'étudier les nouvelles pratiques compétitives appelées e-sport (ou sport numérique) et comment elles transforment la vision de la société et du joueur à propos du jeu. Déjà au début des années 2000, Philippe Mora et Stéphane Héas (2003) 
avaient montré que le rapport au corps est également important pour les joueurs de jeux vidéo. De la sorte, il peut être intéressant d'analyser, en vis-à-vis des sports dits traditionnels avec ces nouvelles pratiques de e-sports qui se situent bien dans des cadres compétitifs, où des joueurs professionnels commencent à émerger, comme le fait Nicolas Besombes (dans ce numéro). Mais, d'autres pistes peuvent également être proposées à partir de trois points de réflexion qui se dégagent du développement de jeux numériques et de leur relation au sport : 1) la mise en scène du sport et ses relations avec un positionnement subjectif et le Gameplay, 2) l'émergence de nouvelles pratiques et d'espace sociaux qualifiés d'e-sport, 3) la place des dispositifs techniques dans les pratiques et la compétition.

\section{Mise en scène numérique du sport et subjectivation de l'expérience}

17 En premier lieu, on peut interroger les manières de mettre en scène le sport dans les jeux numériques. Ce type de jeux connaît un succès important, et parmi les plus connus et les plus rentables figurent les jeux de football tels que la série des Fifa (1994 : Electronic Arts), ou sa concurrente Pro Evolution Soccer (2001: Konami). Leur succès est si important qu'une nouvelle version de ces titres est éditée annuellement. À côté de ces exemples bien connus, on trouve de multiples types de simulations, parmi lesquels des sports d'équipes (basketball, baseball, hockey, volleyball, football américain, etc.), des sports individuels (équitation, golf, tennis, boxe et autres sports de combat, de skateboard), des sports d'hiver, des sports mécaniques (course automobile ou de moto), des compétitions multisports comme des Jeux Olympiques ou championnats d'athlétisme. Dans un autre registre, nous pouvons aussi citer des jeux de management sportif qui ont rencontré un certain succès, tels que Basketball Pro Management (2012 : Umix Studios), Pro Rugby Manager (2014: Cyanide), Pro Cycling Manager (2005: Cyanide) et de nombreux jeux dédiés au management d'une équipe de football dont Football Manager (2004: Sports Interactive). Dans ces différents jeux, les situations sont diverses, le joueur peut être amené à contrôler des sportifs ou bien à jouer le rôle d'entraîneur/manager.

Une fois mis de côté l'opposition jeu/sport ainsi que l'aspect catégoriel des jeux de sports ou de simulation de sport, il nous reste à nous intéresser notamment à la place du joueur face au jeu. En effet, il ne faut pas oublier que dans ces jeux, le joueur est à la fois acteur et spectateur. Présenté à l'écran, le jeu est souvent une simulation d'une retransmission télévisée qui réutilise les codes de ce médium. Notamment, la vue peut-être celle d'une caméra en surplomb, on assiste à des ralentis lorsqu'un point ou un but est marqué ou lorsqu'une faute est constatée, les matchs peuvent faire l'objet de commentaires audio dont les voix sont parfois celles de commentateurs télévisés connus. Comme le dit Brett Hutchins (2008, p. 857), il s'agit « du sport en tant que média [this is sport as media] ». Sur le plan théorique, cette mise en scène renvoie à l'idée de "remédiation » de Jay David Bolter et Richard Grusin (1999, p. 45), c'est-à-dire « la représentation d'un médium dans un autre ». Il faut ajouter à cela que des jeux comme Fifa ou PES offrent la possibilité de jouer avec des équipes connues, comme la Juventus de Turin ou l'Ajax d'Amsterdam, et les personnages sont modélisés à partir de l'apparence physique des joueurs de ces clubs. Certains jeux plus anciens, même s'ils n'allaient pas aussi loin dans la représentation visuelle, aussi bien pour des questions techniques que des questions de droits à l'image ou d'utilisation de patronymes, permettaient déjà de jouer avec des équipes nationales. 
L'évolution vers la situation actuelle montre le poids qu'a pris l'industrie du jeu vidéo en quelques années. Il ressort finalement qu'on ne joue pas ici au foot (comme on ne joue pas au basket aux jeux NBA), mais il semble plutôt qu'on joue, dans ces différents cas, à une mise en scène télévisée du sport. C'est moins une simulation sportive qu'une simulation de médiation sportive, bien que certains voient dans le développement des graphismes en trois dimensions un moyen de développer le réalisme et l'immersion du jeu ${ }^{13}$.

Par contraste, d'autres jeux proposent une mise en situation qui peut être qualifiée de plus «immersive». Dans Wii Sports (2006: Nintendo), le bowling, le golf ou le baseball demandent de tenir le contrôleur (appelé wiimote), d'une façon similaire à l'objet dans le sport concerné (boule, club ou batte). Ils cherchent à simuler la sensorimotricité des activités qu'ils représentent. Ces jeux sont aussi l'héritage de certains jeux d'arcade à la première personne qui proposaient une vue subjective et dont l'invention remonte pratiquement au même moment que les premiers jeux vidéo comme le Shooting Gallery de la Magnavox Odyssey en 1972 (Baer, 2012, p. 30) ou les Beam Gun Games de Gunpei Yokoi chez Nintendo dès 1971 (Takefumi, 2010). Dans ces jeux, le joueur manipule une réplique de pistolet, fusil ou mitrailleuse factice, et sans personnage le représentant, et vise des personnages ou des objets qui apparaissent à l'écran. La relation au dispositif technique de ces jeux implique donc qu'elle soit pensée d'une autre manière qu'en terme de personnage ou d'avatar comme ce qui est généralement le cas (Di Filippo, 2012). En effet, ces exemples nous amènent à questionner les mécanismes d'identification mis en œuvre à travers les dispositifs techniques. Du personnage sélectionné entouré d'un cercle, à la vue subjective, en passant par la vue à la troisième personne (vue de dos), plusieurs types de représentations visuelles existent et donnent lieu à des expériences de jeu variées. Enfin, la mise en scène réaliste du sport n'est pas toujours recherchée par les développeurs. Notamment, des jeux comme Nintendo World Cup (1990: Nintendo) sur NES, NBA Jam (1993 : Midway) sur plusieurs plateformes, ou encore le jeu de boxe Ready to Rumble Boxing (1999, Midway), s'appuient sur un aspect caricatural et sur l'exagération des actions des personnages où l'aspect sensationnel prime sur le réalisme. Le triptyque sport, réalisme et plaisir de jeu est donc à repenser dans le cadre, au moins, de ces jeux.

\section{E-sport : nouveaux espaces sociaux de compétition}

Le deuxième point, qui découle des remarques précédentes, amène à réfléchir à l'émergence de nouveaux modes d'organisation de l'espace social spécifiques aux pratiques ludiques, et plus particulièrement à ce qui est aujourd'hui appelé e-sport ou sport électronique, c'est-à-dire des compétitions organisées autour d'activités vidéoludiques.

21 Les compétitions de jeux vidéo ne sont pas récentes et le phénomène remonte au début des années 70 (Besombes, dans ce numéro), c'est-à-dire relativement tôt dans l'histoire de ce médium. Mais de manière générale, les compétitions restaient des événements épisodiques. Elles étaient souvent utilisées comme moyen marketing. L'e-sport en tant que tel se développe véritablement à la toute fin des années 1990 et au début des années 2000 , notamment grâce aux développements du réseau internet. La compétition de jeu vidéo est caractérisée dès lors par une plus grande régularité, une institutionnalisation des compétitions et la consécration de nouvelles «stars». On voit apparaître des fédérations, parmi lesquelles ont peut citer la Cyberathlete Professional League (CPL) fondée en 1997, à dimension mondiale, et, plus tard, les World Cyber Game (WCG) lancé en 2000 
sous l'impulsion de l'entreprise coréenne Samsung. Ces pratiques se sont développées notamment en Corée du Sud, mais elles ont aujourd'hui un rayonnement mondial ${ }^{14}$. En France, il existe d'ailleurs des équipes de joueurs professionnels comme Millenium ${ }^{15}$. Cette situation offre la possibilité d'une approche socioanthropologique des joueurs professionnels comme l'a fait Jacques Bernard (2003) pour le monde des Échecs. Pour faire le lien avec les épreuves sportives, certaines ligues, comme la WCG, reprennent les codes cérémoniaux propres aux Jeux Olympiques : équipes nationales, médailles, cérémonies d'ouverture et de fermeture, ainsi que des valeurs morales, comme l'idée de fraternité globale (Hutchins, 2008, p. 858). Elles s'en différencient néanmoins par certains aspects : au lieu des diffusions majoritairement faites via la télévision et la radio, elles s'appuient sur la diffusion via le réseau internet et des sites web, les diffusions télévisées restant minoritaires et l'affaire de chaînes de moindre diffusion (GameOne, Gong, Nolife et L'Equipe21).

Tout comme il est nécessaire d'envisager le contexte économique du XIX ${ }^{\mathrm{e}}$ siècle dans le développement des sports traditionnels et leur organisation, il est impératif de tenir compte des systèmes économiques au sein desquels l'e-sport se développe. Dans une optique financière, certains spécialistes du milieu du jeu peuvent proposer des pistes pour que l'e-sport continue à se développer : diversité des jeux, diffusion géographique, régulation plus importante du jeu, management des droits de retransmission médiatiques, et gestion des conflits entre nouveaux médias et médias plus anciens qui répugnent encore à diffuser des compétitions ${ }^{16}$. Ainsi, la chaîne anglaise $\mathrm{BBC}$ va retransmettre les championnats du monde de League of Legends (2009: Riot Games) sur son canal $\mathrm{BBC}$ Three, non pas à la télévision, mais via une partie de son site internet dédiée aux événements sportifs ${ }^{17}$. Cependant, tous les jeux vidéo ne se prêtent pas à la compétition organisée. Les jeux de rôle ou des jeux d'aventures «solo" tout comme des jeux de type survival horror tels que la série des Resident Evil (1996: Capcom), ne conviennent guère à la compétition directe entre joueurs, même si certains youtubeurs vivent très bien des vidéo qu'ils réalisent à partir de ces jeux ${ }^{18}$. Actuellement, les jeux de type arène de bataille en ligne multijoueurs (abrégé MOBA pour multi-player online battle arena) connaissent un succès important (Besombes, dans ce numéro), mais les jeux de tir à la première personne (FPS) ou jeux de stratégie en temps réel (RTS) sont également bien représentés. Étudier les types de jeux utilisés en e-sport permet alors d'interroger la notion de genre dans son articulation avec la dimension compétitive pour éclairer quelques caractéristiques propres à des genres de jeu et leurs implications dans l'établissement de relations sociales : entraînements, progression, présence ou non d'aspect coopératif, plate-forme de préférence et autres dispositifs techniques mis en place (casque et micro pour chat vocal). De l'aspect coopératif va alors découler une forme d'organisation sociale. Il nous semble alors intéressant d'étudier ces différentes formes d'organisation à la lumière de théories sociologiques, par exemple, la sociologie interactionniste d'Howard S. Becker, pour comprendre l'émergence de nouveaux " mondes sociaux » comme le proposent Vincent Berry et Manouk Borzakian (2015). Pour rappel, il s'agit de considérer les productions vidéoludiques comme des œuvres afin « d'examiner les possibilités offertes par la notion de monde de l'art pour une meilleure compréhension des modalités de production et de consommation des œuvres d'art " (Becker, 1988, p. 22). 


\section{Place des dispositifs techniques dans la compétition}

niverut niveaux de difficulté et des obstacles où le principe du high score définit les conditions de compétitions entre différents acteurs (Besombes, dans ce numéro). Dans ces cas, les dispositifs techniques servent de dispositifs de médiation entre différentes personnes individus. Lorsqu'elles mettent plutôt en scène un affrontement entre des individus et des programmes d'intelligence artificielle, les relations peuvent prendre diverses formes. Suivant ce principe, Lisa Rougetet (dans ce numéro) illustre l'articulation entre les recherches dans le domaine de l'intelligence artificielle et la mise en scène de la compétition homme/machine. Plusieurs types de relations entre hommes et machines peuvent de même s'établir. Ces dernières peuvent faire figure d'opposants, comme dans le cas des Échecs, ou servir à mettre en scène des ennemis (monstres) aussi bien que des alliés (PNJ). Il est possible d'aborder cette analyse à l'aune des réflexions de Bruno Latour, pour qui certains objets deviennent des « acteurs non-humains » qu'il regroupe avec les acteurs humains sous le terme, emprunté à Greimas (1967), d'« actants » (Latour, 2005, p. 202). La forme construite et la mise en scène de ces actants participe ainsi à une reconfiguration des mondes sociaux. Ce que l'on appelle l'intelligence artificielle (I. A.) d'un jeu peut constituer un critère de qualité pour certains joueurs, rappelant les recherches pionnières dans ce domaine (Rougetet, dans ce numéro). Nous ajouterons à ces dernières formes d'affrontement, en prenant un peu d'avance sur le paragraphe qui suit le cas de certains games with a purpose (ou gwap ou jeu avec but) comme le jeu français Akinator qui propose d'affronter une intelligence artificielle sous la forme d'un jeu de devinettes qui permet d'alimenter la base de connaissances (c'est le but de ce gwap) du logiciel associé à ce jeu (Lafourcade et al., 2015, p. 102). De même l'affrontement supposé peut être détourné comme dans le cas d'un autre cas de gwap tel que Foldit (voir cidessous) afin de résoudre des problèmes dont ni les concepteurs, ni l'intelligence artificielle, ni les joueurs ne connaissent a priori la réponse (p. 15). La compétition n'a plus lieu entre l'individu et une "machine ", mais elle est transformée en une nouvelle forme de compétition qui confronte l'individu allié à la machine face à un problème d'importance à résoudre. L'étude de cette mise en relation d'un individu face à un dispositif technique dans une optique plus ou moins sérieuse, mérite dès lors le questionnement scientifique (Caulfield et Maj, 2008 ; Lafourcade et Fort, 2014).

\section{Jeux et apprentissage : développement, éducation et rééducation}

De longue date, on a tenté d'adapter ou de détourner des jeux à d'autres fins que l'on qualifie actuellement de sérieuses, la fin la plus courante étant l'éducation des enfants. Le jeu par sa nature a alors pour objectif d'aider à motiver l'enfant et l'accompagner dans sa 
transition entre monde ludique et sérieux. De même, on peut citer les divers travaux consacrant la participation du jeu dans le développement, dont le nombre important de recherches en psychologie comme celles de Freud, Winnicot, Piaget, Vigotski, Chateau, Wallon, etc. (Brougère, 2005, p. 19-37). Dans cette lignée, les approches psychologiques du jeu vidéo s'intéressent d'abord aux jeux chez les mineurs (aussi bien chez les enfants que les adolescents), au risque d'induire un biais de représentation dans les échantillons (Rueff, 2008), laissant de côté la question de l'adulte ou de la maturité de la personne face au jeu. Ceci a notamment pour conséquence une littérature particulièrement fournies à propos de la violence dans les jeux vidéo et de l'apprentissage de la brutalité (Coslin, 2012 ; Romo et al., 2012 ; Fournis et al., 2014) dont les travaux peuvent intéresser le grand public (Bègue, 2012). Ces travaux et débats méritent d'être mis en confrontation avec ceux qui s'étaient précédemment penchés sur des approches hypothétiques relativement proches à propos des comics américains (Wertham, 1953) ou, plus récemment, à propos des dessins animés japonais (Hurard, 1997).

À l'inverse, le jeu peut être considéré en termes d'apports y compris dans le domaine de la santé. En tant que jeu " classique » sous diverses variantes, il est employé à la fois pour aider les personnes âgées à notamment maintenir une activité intellectuelle et sociale (Cloux, 2013). Les maisons de retraite proposent ainsi des activités autour de jeux de cartes, de jeux de tuiles (dominos), de jeux d'adresses, des jeux de société ou de jeux de lettres (Mots Croisés, Mots Mêlés, Pendu, Scrabble, etc.). De la sorte, même des jeux de stratégie abstraits tels que les Échecs (Fried, 1992) ${ }^{19}$ ou le Go (Yasuda, 2003) ${ }^{20}$ peuvent être employés comme outil de communication afin d'inciter certaines personnes à s'exprimer au contact d'autrui. Ces jeux peuvent donc aussi s'analyser en termes de potentiels pour stimuler les personnes aux mêmes titres que les jeux d'adresses ou sportifs, dont la fameuse Balle au camp/prisonnier. Toutefois, il nous faut noter que même si les jeux sportifs sont employés depuis très longtemps dans les écoles dans des cadres d'activités d'Education Physique et Sportive (EPS), les travaux de recherche portant sur les jeux et l'éducation ont tendance à les oublier (Parlebas, 2003). Désormais, c'est plutôt au regard les jeux sérieux que cette comparaison est effectuée. En effet, la littérature comporte déjà de nombreux travaux consacrés à l'intérêt thérapeutique de la Nintendo Wii (Jamal et al, 2011) ou de la Xbox Kinect (Beaulieu-Boire et al., 2015). Les travaux portant sur la thérapie par le jeu (play therapy) employant le jeu classique ou vidéo représentant déjà un domaine de recherche à part entière tellement leur nombre est important (Schaefer, 2011; Crenshaw et al., 2015). Ainsi, le jeu a donc sa véritable place en Santé et en Éducation, alors que dans d'autres milieux professionnels il a encore un peu de mal à s'affirmer. Cela est peut-être dû à l'association sémantique qui est faite entre jeu et loisir.

\section{Jeux, loisirs, travail et sérieux}

Depuis les écrits de Roger Caillois (1958), le jeu est le plus souvent considéré comme un loisir qui participe du temps libre, par opposition au temps contraint du travail. Mais ces catégories n'ont pas toujours été ainsi distinguées, comme le rappelle Paul Yonnet (1999, p. 76). Cette relation ambiguë qui lie et oppose jeu et loisir ou ludique et sérieux a été relevé, il y a déjà quelques dizaines d'années. Johan Huizinga (1951, p. 73) l'a ainsi exprimé : « le sérieux tend à exclure le jeu, tandis que le jeu peut fort bien englober le sérieux ». On peut ainsi jouer sérieusement ou acquérir de nouvelles connaissances en jouant, mais il est plus difficile de considérer qu'une activité sérieuse puisse faire appel au 
jeu. Toutefois, même cette acquisition de connaissances, si elle est admise en contexte d'apprentissage, l'est difficilement en dehors de ce cadre particulier. Il y a ainsi une opposition travail / jeu qui est faite. Il ne s'agit pas seulement de considérer un jeu pour ce qu'il apporte dans la réalisation d'une tâche plus sérieuse, mais aussi d'aborder le jeu en entreprise, même si ce dernier reste fondamentalement ludique. Cette approche du jeu en contexte sérieux relève plutôt de la sociologie du travail ou des sciences de gestion. La pratique du jeu peut elle-même être analysée en termes de pratiques résistancielles ou subversives (Le Lay, 2013), mais aussi être identifiée pour ce qu'elle apporte dans la lutte contre l'ennui et en termes de gains de motivations pour les employés, que cela soit avec des jeux plus traditionnels (Roy, 1959) ou des jeux actuellement surtout numériques (Lejealle, 2008).

28 En dehors de ces champs d'analyse, comme nous l'avons abordé plus haut, le jeu est utilisé de longue date dans des contextes sérieux pour l'apprentissage (Schmoll, dans ce numéro). Même si, comme le rappelle souvent Gilles Brougère (2002, p 7), « en jouant, on apprend avant tout à jouer ", si le jeu est détourné, adapté ou conçu pour une tâche d'apprentissage, ce dernier peut dépasser les limites de la simple acquisition de connaissances liées directement au jeu et à ses mécanismes. Cependant, la relation entre jeu et apprentissage n'est pas à considérer uniquement du point de vue de l'enfant. Les adultes peuvent aussi bénéficier d'un apprentissage par le jeu. C'est d'ailleurs ce point de vue qui est à l'origine du développement des jeux sérieux ou serious games. Pourtant, l'association jeu et loisir continue de poser quelques difficultés y compris aux chercheurs qui y dédient leurs recherches. Il y a encore quelques années « les "sciences du jeu" qui commençaient à se structurer autour de quelques revues en langue anglaise étaient encore en France, un champ de recherches en émergence. Que les recherches soient dédiées aux jeux sérieux ou aux autres formes de jeux, ces études étaient souvent considérées comme futiles, en quelque sorte à l'image de leur objet» (Schmoll, 2011a, p. 11). Plusieurs d'entre nous, d'ailleurs, ont fait cette malheureuse expérience de l'interprétation de la dichotomie chercheur-joueur (Di Filippo, 2012). Aujourd'hui, les choses semblent avoir changé. Nous supposons que ce changement a eu lieu, notamment, suite au développement dans le nord de l'Europe et outre-Atlantique des travaux de recherche du types game studies qui se sont en partie nourris de l'explosion de la pratique des jeux vidéo au cours des années 1990 (Triclot, 2013). Il aura encore fallu quelques années pour que ce changement de perception des recherches sur le jeu soit aussi accepté en France.

\section{Les formes de jeux à des fins sérieuses}

Depuis les années 2000, l'expression «serious game » qui se traduit en français plutôt par l'expression «jeu vidéo sérieux» est usitée dans les littératures scientifique et professionnelle pour parler de certains emplois du jeu à des fins sérieuses. Cependant, il ne s'agit pas de la seule expression employée pour parler de jeux ou du jeu appliquée à des fins sérieuses, mais de la plus répandue. Il nous faut ainsi prendre en compte d'autres formes d'emploi du jeu à des fins sérieuses qui génèrent une littérature scientifique conséquente. Parmi, ces autres conceptions, nous signalons les travaux portant sur le " serious gaming" et le "serious play", les "games with a purpose " (jeux à buts) et la " gamification » (ou ludification). 
Quoi qu'il en soit, ces différents développements et travaux nous incitent à nous interroger d'une manière différente sur les notions de jeux et de sérieux. Dès lors, même si l'expression "serious game ", dans son acception actuelle de jeu conçu et employé à des fins professionnelles, date de 1970 (Abt) ; elle n'a véritablement été employée qu'au début des années 2000 avec pour toile de fond la prise de conscience, d'abord américaine du potentiel offert par les dernières générations de jeux vidéo $0^{21}$. Or, dès l'avènement de cette notion de serious game, de vieux débats datant des travaux de Caillois et de Huizinga ont été remis au goût du jour reprenant la question de la combinaison possible ou non entre jeux et sérieux. Dans cet ordre d'idées, la distinction entre jeu et sérieux doit se faire et est réalisée par l'importance que l'on accorde ou non à la formalisation de l'apprentissage " ou que l'on accepte l'idée que l'apprentissage viendra en plus sans que l'on cherche à le formaliser » (Brougère, 2012, p. 128). Cette distinction se fait surtout du point de vue de la pratique (play) du jeu (game), car même en dehors des cadres d'apprentissages, l'histoire ne manque pas de cas où le jeu a été employé à des fins sérieuses, comme ce que l'on nomme désormais la théorie des jeux (Eber, 2007, p. 6). Si un tel nom lui a été donné, ce n'est pas le fait du hasard. Ce nom est associé à l'observation et l'étude de jeux d'un point de vue mathématique afin de définir « la meilleure stratégie à entre prendre pour gagner à coups sûr ». Du moins, tel était le point de départ de ces travaux. À l'opposé de cette argumentation, pour Julian Alvarez et Damien Djaouti (2012), à la pointe de la recherche française à propos des serious games, la question du jeu et du sérieux ne relève pas du fait que l'on puisse sérieusement jouer à un jeu, mais plutôt que l'on puisse concevoir un jeu dédié à un usage non ludique. En ce sens, ils opposent les notions de serious game et de serious gaming. Selon leur définition, le serious gaming relève d'une attitude sérieuse adoptée face à un jeu y compris le détournement de véritables jeux à des fins sérieuses. On peut encore actuellement s'interroger sur cette opposition, car de nombreux auteurs anglophones qualifient de serious gaming la pratique de serious games dans des organisations (Baek et al., 2014 ; Young et al., 2012). On peut alors préférer à cette expression ambiguë, celle de serious play pour qualifier des emplois détournés de jeux à des fins non purement ludiques (Roos et al., 2004). On pourra d'ailleurs questionner le fait que l'expression serious play se soit développée plus tôt que celle de serious game (Rieber et al., 1998).

\section{Point de vue des concepteurs et des joueurs}

Si l'on se focalise, conformément au point de vue développé par Julian Alvarez et Damien Djaouti (2010, p. 17), sur l'intention du concepteur du jeu pour le qualifier de sérieux ou non, d'autres questions apparaissent. Parmi celles-ci, la question du type d'emploi peut être posée. En effet, les serious games peuvent répondre à trois grandes finalités : " diffuser un message (éducatif, informatif, persuasif, subjectif), dispenser un entraînement (cognitif, physique) » et « favoriser l'échange de données » (p. 28). Nous pouvons ajouter à ces finalités celle de "l'expérimentation de situations » auxquelles le joueur ne pourrait pas se confronter sans risque et celle d'" attraction d'une catégorie spécifique de population » comme celle des adolescents plus enclins à jouer et à être intéressés a priori par quelque chose que l'on appelle jeu (Thevenot, 2010). Du point de vue du concepteur, la finalité fait le serious game ou serious gaming, mais l'atteinte ou non des objectifs assignés au jeu est l'affaire des joueurs et des personnes qui ont été convaincues par l'intérêt de la mise en place du jeu sérieux et donc d'un changement de pratique 
organisationnelle. De même, nous pouvons aussi nous interroger, comme Stéphane Goria (2012), sur les biais conceptuels qu'entraîne un transfert de connaissances à partir d'un jeu qui est, par principe, une simplification de la réalité ainsi que le résultat d'un ensemble de choix de conceptions et de mises en œuvre. Pour ce qui concerne le support du jeu en lui-même, le serious game peut tout aussi bien être développé sous une forme numérique que sous une autre forme telle qu'un jeu de plateau (Michel \& McNamara, 2015) sans que cela pose d'autres questions de transfert de support et de format qui ne seraient pas abordées par les études des jeux non-sérieux. Toutefois, d'un point de vue pratique, une distinction peut tout de même s'effectuer entre jeu sérieux numérique et non numérique. Il s'agit de la facilité de conception et rapidité de mise en pratique. En ce sens, les serious board games (jeux sérieux sur plateau) possèdent un avantage sur leur équivalent numérique, surtout si les phases d'adaptation du jeu sont nombreuses (Lamey \& Bristow, 2015).

32 À l'opposé de la considération du concepteur du serious game, celle du joueur peut-être prise en compte. Ce qui va faire que le jeu est reconnu comme sérieux est intrinsèquement lié au sens que le joueur donne à l'objet "game ». Les questions sousjacentes à ce point de vue relèvent alors des théories de Jacques Henriot $(1989$, p. 300) et Gonzalo Frasca (2007, p. 70) selon lesquelles, c'est l'attitude du joueur face à l'objet qui fait sens. L'une des questions liées relève des éléments qui constituent la reconnaissance d'une chose en tant que jeu. C'est aussi ce que traite l'approche la sémio-pragmatique lorsqu'elle met en avant le rôle du récepteur du «jeu» dans la compréhension, l'actualisation et l'appropriation d'une œuvre, c'est-à-dire de l'objet ludique (Genvo, 2006 ; Kellner, 2007 ; Thevenot, 2015). En ce sens, Sebastien Genvo propose la notion de marqueur de jouabilité et pose la question de leur reconnaissance et évolution sociétale : «nous pouvons avancer que les dispositifs à vocation de jeu doivent également convaincre leur destinataire de leur dimension ludique à travers des "marqueurs pragmatiques", qui répondent à certaines représentations de l'activité et qui évoluent à travers les sociétés. » (Genvo, 2011, p. 70). Même si l'intention du concepteur est de proposer un jeu à son public, c'est le joueur qui fait de l'objet un jeu.

Reste le point de vue des autres personnes associées aux jeux sérieux, mais qui ne sont ni des concepteurs ni des joueurs. Cette question des autres publics du « jeu», comme celle de leur réception $\mathrm{du}$ « jeu » et de la mise en relation avec ce dernier peuvent aussi être posées. Cette problématique se retrouve par exemple dans la conception de jeux sérieux que l'on nomme "games with a purpose ». Il s'agit dans ces cadres de reformuler un problème sous la forme d'un jeu, afin d'inciter, le plus souvent, un maximum de joueurs / contributeurs à participer à la résolution du problème. Les cas d'applications actuellement les plus exploitées concernent l'indexation de documents, la recherche biologique ou médicale et l'amélioration du traitement automatique du langage (Lafourcade et al, 2015). Il est légitime de se poser la question de ce point de vue, si nous sommes face à des objets non-ludiques proposés sous la forme d'un jeu pour faire croire aux joueurs qu'il s'agit bien d'un jeu? (Genvo, 2013, p. 34). Il peut aussi être intéressant de s'interroger sur le positionnement de ce type de jeux par rapport aux autres jeux sérieux. Par exemple, le gwap le plus célèbre, Foldit, pose la même question. Foldit est une sorte de jeu de réflexion qui se présente, par niveau de difficulté croissante, à la manière du jeu Candy Crush Saga (2012: King), des problèmes de pliages de protéines à réaliser au fur et à mesure, dont la solution du dernier niveau est inconnue. Une communauté de joueurs a été ainsi sollicitée pour résoudre un problème de pliage sur lequel les chercheurs en 
biologie médicale bloquaient depuis des années. En quelques semaines, la solution fut trouvée par l'un des 20000 joueurs. Toutefois, si du point de vue de ces concepteurs et joueurs, Foldit est bien un jeu, du point de vue de certains chercheurs, il s'agit plutôt d'un logiciel de modélisation de protéines en 3D qui a été gamifié, c'est-à-dire qu'il est issu d'un logiciel ayant subi un processus de gamification (Deterging, 2012). Bien entendu, cette question peut être évitée si la qualification de « jeu » est laissée aux joueurs. Mais, cette qualification de joueur elle-même peut dépendre de ce que certains qualifieront de jeu et de son contexte. De la sorte, le contexte d'usage du serious game pose aussi question. Estce qu'un environnement sérieux va avoir une influence sur la perception du joueur pour qualifier l'objet de sa pratique sérieuse de jeu ? L'élève, en classe, confronté à l'emploi obligatoire d'un serious game est-il encore en mesure de jouer? Cette question fondamentale pour qui étudie le serious game est centrale dans la contribution de Michel Lavigne (dans ce numéro) qui s'interroge sur la perception ludique des serious games et la frontière entre loisir et apprentissage. Victor Potier (dans ce numéro), quant à lui, étend cette question au fait que le contexte d'usage change avec le jeu, d'autant plus si le jeu est numérique et accessible depuis l'extérieur de l'organisation qui l'accueille. Considéré comme un jeu, le serious game peut-être aussi joué, en dehors de la sphère professionnelle ou d'enseignement, même si sa réception peut être complètement différente.

Enfin, une dernière question autour du jeu et du sérieux peut être posée. Il s'agit de l'impact du support sur le fait qu'un objet puisse être considéré par ses "joueurs " comme un «jeu ». Cette question a déjà été soulevée de nombreuses fois. Elle permet de considérer ce qui peut être admis comme un jeu à partir de son support (Schell, 2010, p. 51) d'envisager ce support en termes d'airs de familles avec d'autres choses reconnues comme des jeux ou non par leur public (Goria, 2014) ou bien de prendre en compte le changement de support (le plus souvent du physique vers le numérique) dans le cadre d'un apprentissage par le jeu tel que Laurence Schmoll l'aborde (dans ce numéro). Il peut d'ailleurs être intéressant de ne pas opposer les jeux par leur support et les considérer comme complémentaires vis-à-vis d'un contexte sérieux. Une analyse transmédiatique de jeux aux mêmes objectifs sérieux peut être riche en informations, telle que celle réalisée à propos des jeux modélisant les changements climatiques (Reckien \& Eisenack, 2013).

\section{Conclusion}

Les jeux numériques comme les jeux traditionnels peuvent s'analyser et être interrogés selon une variété considérable de points de vue. Si notre interrogation de départ nous faisait craindre une prise de pouvoir des jeux numériques sur les autres, cela n'est pas établi. Toutefois, face à la multitude de travaux dédiés exclusivement aux jeux numériques, le chercheur doit faire l'effort de regarder ce qui se fait du côté des jeux traditionnels et ce, quel que soit le contexte d'observation à considérer. Dans le cas contraire, le paysage de la recherche sur les jeux lui semblera, hors perspective purement historique, uniquement relever des jeux informatisés.

Il y a ainsi quelques points à retenir de ce parcours de la littérature identifiée en cherchant à considérer la variété des supports possibles. Il ne faut donc pas déduire trop rapidement que les jeux numériques viennent remplacer les jeux non-numériques ou ne les inspirent que dans un sens. Il existe de très nombreuses influences réciproques et entrecroisements entre ces deux grandes catégories de jeux. Cela doit inviter à les penser conjointement et pas seulement dans le cadre de l'étude de jeux hybrides. Il nous semble 
aussi nécessaire de réinterroger les catégories d'usage à la lumière de différents types de jeux caractérisés, notamment, par leur différence de support. De la sorte, il nous parait important de repenser les frontières communément admises entre ces types de jeux ou entre le monde «normal » et les espaces de jeux qu'ils soient sérieux ou non. Les jeux ne sont pas un risque pour la société, ils s'adaptent à celle-ci.

\section{BIBLIOGRAPHIE}

ALVAREZ J. \& DJAOUTI D. (2012), Introduction au Serious Game, Paris, Questions Théoriques.

ARCHIBALD S. \& GERVAIS B. (2006), « Le récit en jeu: Narrativité et interactivité », Protée, vol. 34, n 2-3, pp. 27-29. Accès : http://www.erudit.org/revue/pr/2006/v34/n2-3/014263ar.pdf

ARSENAULT D. \& MAUGER V. (2012), «Au-delà de "l'envie cinématographique" : le complexe transmédiatique d'Assassin's Creed ", Nouvelles vues. Revue sur les pratiques et les théories du cinéma au Québec, $\mathrm{n}^{\circ}$ 13. Accès : http://www.nouvellesvues.ulaval.ca/futur-numero/no-13-le-cinemaquebecois-et-les-autres-arts-dirige-par-elspeth-tulloch/articles/au-dela-de-lenviecinematographique-le-complexe-transmediatique-dassassins-creed-par-dominic-arsenault-etvincent-mauger/

BAEK Y., KO R. \& MARSH T. (2014), Trends and Applications of Serious Gaming and Social Media, Berlin, Springer.

BAER R. (2012), Ralph Baer: Mémoires du père des jeux vidéo, Cergy, Pixn'Love Editions.

BEAULIEU-BOIRE L., BELZILE-LACHAPELLE S., BLANCHETTE A., DESMARAIS P. O. \&

LAMONTAGNE-MONTMINY L. (2015), «Balance Rehabilitation using Xbox Kinect among an Elderly Population: A Pilot Study ", J Nov Physiother, vol. 5, n 261, pp. 1-5. Accès : http:// www.omicsgroup.org/journals/balance-rehabilitation-using-xbox-kinect-among-an-elderlypopulationa-pilot-study-2165-7025-1000261.php?aid=50099

BEGUE L. (2011), «Jeux video : l'école de la violence», Les racines de la violence, L'Essentiel Cerveau et Psycho, $\mathrm{n}^{\circ}$ 8, pp. 14-21.

BLANCHET A. (2015), « Penser les relations entre cinéma et jeux vidéo à travers la pratique industrielle de l'adaptation », In Brougère G. (dir.), Penser le jeu: Les industries culturelles face au jeu, Paris, Nouveau Monde Éditions, pp. 69-82.

BERNARD J. (2003), « Un ensemble né d'une pratique ludique. Les joueurs d'échecs professionnels ", Socio-anthropologie, $\mathrm{n}^{\circ} 13$. Accès : https://socio-anthropologie.revues.org/175

BERRY V. (2012), L'expérience virtuelle. Jouer, vivre, apprendre dans un jeu vidéo, Rennes, Presses universitaires de Rennes.

BERRY V. \& BORZAKIAN M. (2015), « Introduction », RESET, n 4. Accès: http:// reset.revues.org/541

BOURDAA M. (2012), « Le transmédia : entre narration augmentée et logiques immersives », Inaglobal, Accès : http://www.inaglobal.fr/numerique/article/le-transmedia-entre-narrationaugmentee-et-logiques-immersives. 
BROUGERE G. (2002), « L'enfant et la culture ludique », Spirale, vol. 4, n²4, pp. 25-38. Accès : http://www.cairn.info/revue-spirale-2002-4-page-25.htm

BROUGERE G. (2005), Jouer/Apprendre, Paris, Economica.

BROUGERE G. (2008), « La ronde de la culture enfantine de masse ». In G. Brougère (dir) La ronde des jeux et des jouets. Harry, Pikachu, Superman et les autres, Paris, Autrement.

BROUGERE G. (2012), « Le jeu peut-il être sérieux? Revisiter Jouer/Apprendre en temps de serious game », Australian Journal of French Studies, vol. 49, n², pp. 117-129. Accès: http://www.univparis13.fr/experice/wp-content/uploads/2015/02/Le_jeu_peut-il_tre_srieux__.pdf

BOWMAN S. L. (2010), The Functions of Role-Playing Games: How Participants Create Community, Solve Problems and Explore Identity, Londres, McFarland \& Company.

CAILLOIS R. (1958), Les jeux et les hommes, Paris, Gallimard.

CAÏRA O. (2007), Jeux de rôle : Les forges de la fiction, Paris, CNRS Editions.

CAULFIELD C. \& MAJ S. P. (2008), « Come Play », In Iskander M. (éd.), Innovative Techniques in Instruction Technology, E-learning, E-assessment, and Education,

Dordrecht, Springer, pp. 86-91.

CAYRES V. (2015), « Analise dramaturgica de um universo em expansao: um estudo de World of Warcraft », Passages de Paris, $\mathrm{n}^{\circ}$ 10, pp. 209-222.

CLOUX S. (2013), «Jeu et maladie d'Alzheimer », VST-Vie sociale et traitements, vol. 120, $\mathrm{n}^{\circ}$ 4, pp. 97-101.

CONSALVO M. (2011), « MOOs to MMOs: The internet and Virtual Worlds », In Consalvo M., Ess C. (éd.), The handbook of internet studies, Malden, MA, Oxford, Wiley-Blackwell, pp. 326-347.

COSLIN P. G. (2012), Jeux dangereux, jeunes en danger, Paris, Armand Colin.

CRENSHAW D. A., BROOKS R. \& GOLDSTEIN S. (2015), Play Therapy Interventions to Enhance Resilience, New York, Guilford Publications.

CUVELIER P. (2011), « Jeu et fiction dans un jeu de cartes à collectionner : le cas de Magic :

l'Assemblée ", Strence, ${ }^{\circ} 2$. Accès : https://strenae.revues.org/305

DETERDING S. (2012), « Gamification: designing for motivation », Interactions, vol. 19, n 4, pp. 14-17.

DI FILIPPO L. (2012), « Les notions de personnage-joueur et Roleplay pour l'étude de l'identité dans les MMORPG », Interrogations, n¹5. Accès : http://www.revue-interrogations.org/Lesnotions-de-personnage-joueur

DI FILIPPO L. (2014), « Contextualiser les théories du jeu de Johan Huizinga et Roger Caillois », Questions de communication, $\mathrm{n}^{\circ} 25$, pp. 281-308. Accès : https://hal.archives-ouvertes.fr/ hal-01227855/document

DI FILIPPO L. (à paraître 2016), « MMORPG as Locally Realized Worlds of Action », In Boni M., Lefebvre M., Steinberg M. (éd.), World Building. Transmedia, Fans, Industries, Amsterdam, Amsterdam University Press.

EBER N. (2007), Théorie des jeux, Paris, Dunod.

ELADHARI M., \& LINDLEY C. A. (2003), « Player Character Design Facilitating Emotional Depth in MMORPGs ", Proceedings of the DIGRA Conference (Digital Games Research), pp. 1-14.

ESCOFIER J. P. (2008), Histoire des Mathématiques, Paris, Dunod. 
FOURNIS G., NABHAN-ABOU N., ORSAT M., BRIERE M., LHUILLIER J. P., GARRE J. B. \& GOHIER B. (2015), « Violence, crimes et jeux vidéo violents: le point sur la question », L'information psychiatrique, vol. $91, \mathrm{n}^{\circ} 4$, pp. 331-337.

FRASCA G. (2001), Videogames of the oppressed: videogames as a mean for critical thinking and debate, Master Thesis, Georgia Institute of technology.

FRASCA G. (2007), Play the Message, Thèse de doctorat en Philosophie, IT University of Copenhagen.

FRIED S. (1992), « Chess: A psychoanalytic tool in the treatment of children », International Journal of Play Therapy, vol. 1, n 1, pp. 43-51.

GENVO S. (dir.) (2006), Le game design de jeux vidéo : Approches de l'expression vidéoludique, Paris, L'Harmattan.

GENVO S. (2011), « Penser les phénomènes de « ludicisation » du numérique pour une théorie de la jouabilité », Revue des Sciences Sociales, n 45, pp. 68-77. Accès : http://www.ludologique.com/ publis/Ludicisation_Genvo_S.pdf

GENVO S. (2013), Penser la formation et les évolutions du jeu sur support numérique, Mémoire pour l'habilitation à diriger des recherches, Université de Lorraine.

GORIA S. (2012), « Wargames et stratégies de communication », Communication \& Organisation, $\mathrm{n}^{\circ}$ 42, pp. 133-146.Accès : https://communicationorganisation.revues.org/3903

GORIA S. (2014), « La question du public et de la nature de l'emploi du jeu à des fins sérieuses. Une réflexion développée dans un cadre d'enseignement », Interfaces Numériques, vol. 3, n 3, pp. 521-539.

GOUZOUAZI J. \& TOSSE Y. D. (2015), « "Et Sci ... les savants avaient eu un blog ?" Monter un atelier ludique et participatif d'histoire des sciences en lycée ", Actes des sessions du colloque Sciences \& You, Nancy, Université de lorraine, pp. 261-265. Accès : http://www.science-andyou.com/sites/science-and-you.com/files/users/sy2015_sessions_proceedings.pdf

GREIMAS A. J. (1967), La structure des actants du récit: essai d'approche générative, Word, vol. 23, n¹-3, pp. 221-238. Accès : http://www.tandfonline.com/doi/ pdf/10.1080/00437956.1967.11435478

HEAS S. \& MORA P. (2003), « Du joueur de jeux vidéo à l'e-sportif : vers un professionnalisme florissant de l'élite? », In Roustan M. (dir.), La pratique du jeu vidéo : réalité ou virtualité ?, Paris, L'Harmattan, pp. 131-156.

HENRIOT J. (1969), Le jeu, Paris, Presses universitaires de France.

HENRIOT J. (1989), Sous couleur de jouer, Paris, José Corti.

HINEBAUGH J. (2009), A board game education, Lanham, Rowman \& Littlefield Publishing group. HUIZINGA J. (1951), Homo ludens : Essai sur la fonction sociale du jeu, Paris, Gallimard.

HURARD F. (1997), « L'action des autorités de régulation de l'audiovisuel face à la violence : Le cas de la France ", In Lacroix J.-M. (éd.), Violence et télévision: autour de l'exemple canadien, Paris, Presse de la Sorbonne Nouvelle, pp. 13-30.

JAMAL K., LE FLOCH F \& GALLIEN, P. (2011), « Intérêt de la Wii Fit dans la rééducation de l'équilibre debout ", Kinésithérapie la revue, n 117, pp. 42-46. Accès : https://

www.researchgate.net/

publication/257699996_Interet_de_la_Wii_Fit_dans_la_reeducation_de_l'equilibre_debout_Interest_of_the_Wii_Fit_in_the_upright_ba 
KELLNER C. (2007), Les cédéroms, pour jouer ou pour apprendre?, Paris, L'Harmattan.

LAFOURCADE M., LE BRUN N. \& JOUBERT A. (2015), Jeux et intelligence collective-résolution de problèmes et acquisition de données sur le Web, ISTE éditions.

LAFOURCADE M. \& FORT K. (2014), « Propa-L: a Semantic Filtering Service from a Lexical Network Created using Games With A Purpose », Proceedings of the Ninth International Conference on Language Resources and Evaluation (LREC), Reyjkjavik, pp. 1676-1681.

LAMEY B. \& BRISTOW C. (2015), « The Game as a Talking Tool: Using a Board Game for Creative Consultation, Engagement and Inclusion », In Brewer G. \& Hogarth R. (dir.), Creative Education, Teaching and Learning: Creativity, Engagement and the Student Experience, Basingstoke, New York, Palgrave Macmillan, pp. 243-253.

LATOUR B. (2005 [1989]), La science en action. Introduction à la sociologie des sciences, Paris, La Découverte.

LE LAY, S. (2013), « Des pratiques ouvrières ludiques aux dispositifs managériaux ludistes: vers une instrumentalisation du jeu dans le travail ", La nouvelle revue du travail, $n^{\circ} 2$. Accès : https:// nrt.revues.org/702

LEJEALLE C. (2008), « Jouer sur son mobile: entre respect et résistances aux règles prescrites », Actes du 1er colloque international «Consommation et résistance(s) des consommateurs ", 28 novembre 2008, IRG Université de Paris 12. Accès : https://hal.archives-ouvertes.fr/file/index/ docid/345327/filename/ jouer_sur_son_mobile_entre_respect_et_resistance_aux_regles_prescrites.pdf LESSARD J., \& THERRIEN C. (2015), Evolution des jeux : catalyseurs, mécanismes et agents, Sciences du jeu, $\mathrm{n}^{\circ} 4$, pp. 1-4. Accès : https://sdj.revues.org/pdf/471

MICHEL H. \& MC NAMARA P. (2015), « Serious Games: Faites vos jeux! », Systèmes d'information \& management, vol. 19, $\mathrm{n}^{\circ} 3$, pp. 3-8. Accès : https://www.cairn.info/revue-systemes-d-informationet-management-2014-3-page-3.htm

PARLEBAS P. (2003), « Le destin des jeux : Héritage et filiation », Socio-anthropologie, $n^{\circ}$ 13, pp. 1-9. Accès : https://socio-anthropologie.revues.org/173

PARLEBAS P. (2005), « L'éducation par le sport », Vers l'éducation nouvelle, n517, pp. 70-83.

RECKIEN D. \& EISENACK K. (2013), « Climate change gaming on board and screen: A review, Simulation \& Gaming ", vol. XX, n X, pp. 1-19. Accès : http://nexus.hs-bremerhaven.de/ library.nsf/0946dbe6a3c341e8c12570860044165f/03f7dfba7187d897c1257b5800303d28/\$FILE/ Simulation\%20Gaming-2013-Reckien-1046878113480867.pdf

REY A. (2012), « Loisir », In Rey A. (dir.), Dictionnaire historique de la langue française, pp. 1113.

RIEBER L. P ., SMITH L., \& NOAH D. (1998), « The value of serious play », Educational Technology, vol. 38, $\mathrm{n}^{\circ}$ 6, pp. 29-37. Accès: http://clearwaterschool.com/?page_id=663

ROGERSON M. J., GIBBS M. \& SMITH W. (2015), « Digitising Boardgames: issues and Tensions », Proceedings of the 2015 DiGRA International Conference Digital Games Research Association, May, 2015 vol. 12, pp. 1-16.

ROMO L., BIOULAC S., MICHEL G. \& KERN L. (2012), La dépendance aux jeux vidéo et à l'Internet, Paris, Dunod.

ROOS J., BART V. \& STATLER M. (2004), « Playing seriously with strategy », Long Range Planning, vol. $37, \mathrm{n}^{\circ} 6$, pp. 549-568. 
ROY D. ([1959] 2006), « L'heure de la banane », In Donald R., Un sociologue à l'usine, Paris, La Découverte, pp. 155-187.

RUEFF J. (2008), « Où en sont les « gamestudies » ? ", Réseaux, n 151, pp. 139-166. Accès : http:// www.cairn.info/revue-reseaux-2008-5-page-139.htm

SABIN P. (2012), Simulating war: studying conflict through simulation games, Londres, Continuum International Publishing Group.

SALEN K. \& ZIMMERMAN E. (2003), Rules of Play: Game Design Fundamentals, Cambridge, MIT Press.

SCHÄDLER U. (2007), Jeux de l'humanité : 5000 ans d'histoire culturelle des jeux de société, Genève, Slatkine.

SCHAEFER C. E. (2011), Foundations of play therapy, Hoboken, John Wiley \& Sons.

SCHELL J. (2010), L'Art du game design:100 objectifs pour mieux concevoir vos jeux, Paris, Pearson Education France.

SCHMOLL P. (2007), Chasseurs de trésors. Socio-ethnographie d'une communauté virtuelle. Strasbourg, Néothèque.

SCHMOLL P. (2011a), « Sciences du jeu : état des lieux et perspectives », Revue des sciences sociales, $\mathrm{n}^{\circ}$ 45, pp. 10-19. Accès : http://www.patrick-schmoll.com/pdf/2011-SciencesDuJeu.pdf

SCHMOLL P. (2011b), "Jeux sérieux: exploration d'un oxymore », Revue des sciences sociales, $\mathrm{n}^{\circ} 45$, pp. 158-167. Accès : http://www.patrick-schmoll.com/pdf/2011-JeuxSerieux.pdf

TAKEFUMI M. (2010), Gunpei Yokoi - Vie et philosophie du dieu des jouets Nintendo, Châtillon, Pix'n Love.

THEVENOT P. (2010), Les serious games informatifs : au-delà du jeu..., Mémoire de master en SIC, Nancy-Université.

THEVENOT P. (2015), Nouveauté technologique et milieu professionnel : la tablette tactile et les acteurs de l'immobilier, Thèse de Doctorat en Sciences de l'information et de la Communication, Université de Lorraine.

TRÉMEL L. (2001), Jeux de rôles, jeux vidéo, multimédia, les faiseurs de mondes, Paris, Presses universitaires de France.

TRICLOT M. (2013), « Game studies ou études du play? Une lecture croisée de Jacques Henriot et de Jesper Juul », Sciences du jeu, $\mathrm{n}^{\circ} 1$. Accès : https://sdj.revues.org/223

TRICLOT, M. (2012). Jouer au laboratoire. Le jeu vidéo à l'université (1962-1979). Réseaux, (3), 177-205. Accès : http://data0.eklablog.com/ae-editions/perso/bibliotheque - pdf/triclot philosophie des jeux video.pdf

VEYNE P. (1987), « Olympie dans l'Antiquité », Esprit, n 4, pp. 53-62.

VIEILLE MARCHISET G. \& WENDLING T. (2010), « Aux frontières du sport », ethnographiques, $\mathrm{n}^{\circ} 20$. Accès : http://www.ethnographiques.org/2010/Vieille-Marchiset,Wendling

WENDLING T. (2002), Ethnologie des joueurs d'échecs, Paris, Presses universitaires de France.

WERNER M. \& ZIMMERMANN B. (2003), « Penser l'histoire croisée : entre empirie et réflexivité », Annales. Histoire, Sciences Sociales, $n^{\circ}$ 2003/1, pp. 7-36. Accès : http://www.cairn.info/revueannales-2003-1-page-7.htm

WERTHAM F. (1953), Seduction of the Innocent, New York, Rinehart. 
WOLF M. J. P. (2008), The Video Game Explosion. A History from Pong to PlayStation and Beyond, Westport, Connecticut / Londres, Greenwood Press.

YASUDA Y. (2003), Le Go : un outil de communication, Paris, Chiron.

YONNET P. (1999), Travail, loisir. Temps libre et lien social, Paris, Gallimard.

YOUNG M. F., SLOTA S., CUTTER A. B., JALETTE G., MULLIN G., LAI B., SIMEONI Z., TRAN M. \& YUKHYMENKO M. (2012), « Our Princess Is in Another Castle: A Review of Trends in Serious Gaming for Education », Review of Educational Research, vol. 82, $\mathrm{n}^{\circ} 1$, pp. 61-89.

ZABBAN V. (2012), « Retour sur les gamestudies. Comprendre et dépasser les approches formelles et culturelles du jeu vidéo ", Réseaux, n 173-174, pp. 137-176. Accès : https://www.cairn.info/ article.php?ID_ARTICLE=RES_173_0137

ZYDA M. (2005), « From visual simulation to virtual reality to games », Computer, vol. 38 n $^{\circ}$ 9, pp. 25-32.

\section{NOTES}

1. Pour l'essentiel et avec une écrasante majorité, les jeux numériques sont des jeux vidéo, mais comme les jeux vidéo impliquent l'utilisation d'un écran, cet ensemble de jeux ne représente pas tous les jeux informatiques comme par exemple Blind Legend (2015 : Dowino). C'est pourquoi, nous préférons employer ici l'expression «jeux numériques» moins restrictive que celle de « jeux vidéo ».

2. Pour les jeux qui ont été édités, nous signalons à leur suite et entre parenthèses, la première année d'édition et l'éditeur principal.

3. Dans ce jeu, il s'agit de poursuivre et de rattraper l'autre joueur symbolisé par un rectangle blanc.

4. Premier jeu vidéo (créé par Ralph Baer) reconnu juridiquement dans un procès où il a été confronté à Tennis for Two et Spacewar! (Baer, 2012, 28).

5. En 1952, A.S. Douglas a ainsi programmer le premier jeu vidéo de morpion avec un affichage sur écran d'ordinateur, même si ce dernier était un ordinateur qui n'avait rien de grand public (Baer, 2012, 32).

6. http://www.senscritique.com/groupe/Des_chiffres_et_des_lettres/4708, consulté le 09/02/2016.

7. http://rue89.nouvelobs.com/2015/04/25/realite-alternee-jeu-video-sort-lecrandescend-rue-258817, consulté le 07/10/2015.

8. http://www.monopolypedia.fr/editions/usaopoly/zelda/zelda.php, consulté le 05/10/2015.

9. Ce personnage devient vraiment célèbre avec cette licence, même si c'est déjà le héros de Donkey Kong (1981 : Nintendo).

10. http://www.virtuajdr.net/, consulté le 09/10/2015.

11. http://www.epawn.fr/use-cases-2/, consulté le 09/10/2015.

12. On peut également évoquer les différences entre les concours olympiques de la Grèce antique et les jeux Olympiques tels que les met en place Pierre de Coubertin au XIX ${ }^{\mathrm{e}}$ siècle (Veyne, 1987). 
13. http://www.jeuxvideo.com/videos/chroniques/445193/l-histoire-du-jeu-video-lesjeux-de-sport-a-l-ere-de-la-3d.htm, consulté le 09/10/2015.

14. Un documentaire leur est consacré http://www.millenium.org/home/accueil/video/ game-fever-decouvrez-le-film-documentaire-et-la-nomad-serie-106704, consulté le 22/09/2015.

15. http://www.millenium.org/, consulté le 23/09/2015

16. http://www.gamesindustry.biz/articles/2015-09-30-five-factors-key-to-makingesports-a-usd1bn-business-by-2020?

utm_source=newsletter\&utm_medium=email\&utm_campaign=european-daily, consulté le 02/10/2015.

17. http://www.millenium.org/lol/accueil/actualites/la-bbc-retransmettra-les-worldsleague-of-legends-londres-quarts-de-finale-bbc-three-championnat-du-monde-135248, consulté le 09/10/2015.

18. Par exemple, Pewdiepie a fait plusieurs millions de dollars l'an dernier en réalisant des vidéo où il joue à des jeux du type survival horror tout en tenant le rôle de commentateur (mode Let's Play).

19. avec des enfants

20. avec divers publics constitués d'enfants, de personnes âgées ou handicapées.

21. La Serious Game Initiative lancée aux USA en 2002 peut de ce point de vue être considérée comme l'un des points de départ de la prise de conscience internationale du potentiel des jeux vidéo pour contribuer à perfectionner des techniques professionnelles.

\section{RÉSUMÉS}

Une sorte de paradoxe est en train de s'affirmer à travers la littérature scientifique dédiée aux domaines ludiques ou en rapport avec le jeu. Le nombre de travaux uniquement consacrés aux jeux numériques occulte petit à petit les travaux dédiés aux jeux traditionnels, alors qu'il existe toujours des liens puissants entre jeux traditionnels et jeux vidéo. Un éclairage réciproque devrait nous inciter à nous interroger sur les singularités ou complémentarités des deux domaines, c'est pourquoi nous proposons ici de nous intéresser à certains de ces liens et contrastes existants entre ces deux types de jeux. Cette introduction à ce numéro de Sciences du jeu vise ainsi à montrer une variété de problématiques qui entourent ces phénomènes et comment les articles contribuent à répondre à certains questionnements ouverts ici et proposent des perspectives contemporaines pour l'analyse des jeux de tous genres.

Progressively, a kind of paradox is created through the scientific literature related to the game and play fields. The number of works dedicated only to video games conceals gradually the works dedicated to traditional games, although there are always strong links between those two kinds of games. It seems thus clear that a mutual lighting should make us wonder about the singularity or complementarities of the two areas. We propose to look at some of these existing links and contrasts between traditional and digital games. To this end, the introduction to this issue of Sciences $d u$ jeu will show a variety of research questions surrounding these phenomena. We will 
show how the articles help to address some open questions and offer contemporary perspectives for the analysis of games of all categories.

INDEX

Mots-clés : jeu vidéo, jeu de société, serious games, jouabilité, pratiques ludiques, e-sport

Keywords : video game, tabletop game, board game, serious games, gameplay, play, e-sport

\section{AUTEURS}

DELPHINE BUZY-CHRISTMANN

Centre de recherche sur les médiations, Université de Lorraine

\section{LAURENT DI FILIPPO}

Centre de recherche sur les médiations, Université de Lorraine et Université de Bâle

STÉPHANE GORIA

Centre de recherche sur les médiations, Université de Lorraine

\section{PAULINE THÉVENOT}

Centre de recherche sur les médiations, Université de Lorraine 